\title{
Crecimiento radial de Abies pinsapo en el sur de Chile: relaciones con el clima local y su comparación con poblaciones naturales en España
}

\author{
Radial growth of Abies pinsapo in southern Chile: \\ relationships with the local climate and its comparison with natural populations in Spain
}

\author{
Luis Cardalliaguet a , Ariel A Muñoz*a, Víctor Humanes a , Isabella Aguilera-Betti a,b, \\ Mar Génova c, Carlos LeQuesne d, Moisés Rojas-Badilla ${ }^{\text {a,d }}$, Camilo Veas a

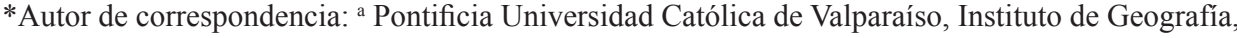 \\ Laboratorio de Dendrocronología y Estudios Ambientales, Avenida Brasil 2241, Valparaíso, Chile, \\ tel.: 56-32-2274082, ariel.munoz@pucv.cl \\ ${ }^{\mathrm{b}}$ Universidad Austral de Chile, Centro Transdisciplinario de Estudios Ambientales y Desarrollo Humano Sostenible (CEAM), \\ Valdivia, Chile. \\ ${ }^{\mathrm{c}}$ Universidad Politécnica de Madrid, Departamento de Sistemas y Recursos Naturales, Madrid, España. \\ ${ }^{\mathrm{d}}$ Universidad Austral de Chile, Instituto de Conservación, Biodiversidad y Territorio, Valdivia, Chile.
}

\section{SUMMARY}

The relationship between the growth of 43-year old specimens of an Abies pinsapo Boiss stand located in the city of Valdivia (Chile) and the climatic variability of this site was studied. The objectives were to define the climatic response function and identify the climatic variables and periods that regulate radial growth rates and to compare the annual radial growth with those characterizing two natural stands of the same species growing in Spain provinces, which, together with Morocco, shelters its last populations. Growth rates in Valdivia, Chile, were similar to or even higher than those in their natural habitat, showing a predilection for warm summers with little rain, as well as for abundant winter precipitation. In relation to the early wood (EW) and late wood (LW) series, the first one was related with prevalent dominant climatic conditions of the previous summer. This may be related to stored food. Late wood showed a climatic signal controlled by dominant conditions of the current growing season. These results indicate that the temperate-rainy climate in Los Ríos Region (Chile), milder than the mountain climate in its original habitat, favors the growth of this species and therefore constitutes an alternative habitat for its conservation. Here, summer temperatures favor its growth, differing from its natural habitat, where soil-water availability is the major factor controlling its growth. This information can be relevant for ex-situ conservation of one of the most severely threatened conifers by climate change and human pressure in the south of the Iberian Peninsula.

Key words: dendrochronology, climate, Abies pinsapo, Spain, Chile.

\section{RESUMEN}

Se estudió la relación entre el crecimiento de un rodal de Abies pinsapo de 43 años de edad cercano a la ciudad de Valdivia, Chile, y la variabilidad del clima local. Los objetivos fueron identificar los factores y periodos climáticos que regulan su crecimiento radial, comparando paralelamente sus tasas de crecimiento con dos rodales naturales de la especie en España, país que junto a Marruecos alberga sus últimas poblaciones. Las tasas de crecimiento en Valdivia fueron similares o superiores a los sitios de su hábitat natural, favorecidas en Chile por veranos cálidos y poco lluviosos, así como por abundantes precipitaciones invernales. A nivel de madera temprana y tardía, la primera se relacionó con las condiciones de los veranos previos, demostrando su relación con sustancias de reserva, mientras que la madera tardía, presentó una señal climática dominada por características de la estación de crecimiento corriente. Estos resultados indican que el clima templado-lluvioso de la región de Los Ríos, menos extremo que el clima de montaña de su hábitat natural, favorece el crecimiento de la especie constituyendo un hábitat alternativo para su conservación. Aquí la temperatura de verano favorece su crecimiento, a diferencia de su hábitat natural, donde el déficit hídrico es el factor que controla su crecimiento. Esta información puede ser relevante para la conservación ex-situ de una de las coníferas más amenazadas por el cambio climático y la presión humana en el sur de la península ibérica.

Palabras clave: dendrocronología, clima, Abies pinsapo, España, Chile. 


\section{INTRODUCCIÓN}

Abies pinsapo Boiss., comúnmente llamado pinsapo, es una conífera perteneciente a los abetos meridionales de Europa, cuyo estado de conservación se clasifica como "en peligro" según la UICN (Linares y Carreira 2006). Relegada a las sierras meridionales béticas del sur de España y a zonas puntuales de la cordillera del Rif para la subespecie marroquí, A. pinsapo ocupa una reducida extensión $c a$. 6.500 ha (Soto et al. 2001), distribuida entre 1.000 y 1.700 $\mathrm{m}$ de altitud, y preferentemente en las laderas de umbría con orientación norte (Linares et al. 2006). Hallazgos de polen fósil y modelos de distribución potencial sugieren que, en el Último Máximo Glacial ( $c a .20 .000$ años atrás) la especie gozó de una distribución más amplia que la actual (Alba-Sánchez et al. 2010). La reducción de precipitaciones que se inició durante el Plioceno y que ocasionó la diferenciación de los abetos mediterráneos, entre ellos Abies alba Mill. y A. pinsapo, de su antepasado común hace 5,33 millones de años (M.a)., continuó hasta el Cuaternario relegando a estas especies a las montañas, en donde las precipitaciones son mayores, constituyendo su refugio hasta nuestros días (Alba-Sánchez et al. 2010). Además del clima, el ser humano ha sido un factor clave para el retroceso del pinsapo y la reducción de su distribución original durante los últimos siglos: el sobrepastoreo, el aprovechamiento para madera o los incendios recurrentes son algunas de las causas que han provocado su disminución (Latorre y Artero 2002). En efecto, el factor antrópico resulta relevante para explicar por qué $A$. pinsapo no ocupa toda el área de distribución potencial derivada de modelos de distribución para la especie (Alba-Sánchez et al. 2010).

En la actualidad, las características climáticas del hábitat natural de $A$. pinsapo son específicas y únicas en la península ibérica. Las sierras béticas en donde se encuentra (sierra Bermeja, sierra de las Nieves en Málaga y sierra de Grazalema en Cádiz) presentan un subtipo particular de clima mediterráneo muy lluvioso (Génova 2007) pudiendo alcanzar $2.000 \mathrm{~mm}$ anuales, aunque con la aridez estival típica del resto de las áreas mediterráneas, que el pinsapo soporta gracias a su carácter xerófilo (respecto de otros abetos) y a su preferencia por zonas umbrías, donde las condiciones no son tan extremas y el efecto del estiaje se amortigua.

Las predicciones climáticas futuras para la mitad sur de la península ibérica infieren una drástica disminución de las precipitaciones, ocasionada por la fase positiva de la Oscilación del Atlántico Norte (NAO), atribuida a los gases de efecto invernadero (Pérez y Boscolo 2010). Los registros del último siglo de la estación meteorológica de Grazalema muestran una tendencia a la disminución de las precipitaciones (figura 1), con episodios secos extremos al final del siglo XX, como el del quinquenio 1991-1995 (Génova 2013). Este escenario futuro podría aumentar la vulnerabilidad y poner en riesgo la supervivencia de los últimos bosques relictos de esta especie en el largo plazo.
En este contexto, el cultivo y la evaluación de plantaciones de la especie en distintas zonas geográficas y condiciones ambientales, pueden ser de gran utilidad para comprender la potencial respuesta futura de la especie en su hábitat natural y para promover su conservación ex-situ.

El presente trabajo describe y analiza los patrones de crecimiento de una plantación de más de cuatro décadas de $A$. pinsapo ubicada en el arboreto de la Universidad Austral de Chile en Valdivia, Chile, y los compara con los de pinsapos creciendo en dos sitios dentro de su hábitat natural en España.

La vulnerabilidad de los pinsapares en su hábitat natural por la amenaza del cambio global (Linares et al. 2013) por un lado, y el aparente rápido crecimiento observado en los ejemplares chilenos, por otro lado, han motivado la realización de este estudio y la posterior discusión acerca de la viabilidad de la conservación de la especie ex-situ. Por este motivo, los principales objetivos del presente trabajo son evaluar las tasas de crecimiento radial de A. pinsapo en Valdivia, con respecto a las tasas de crecimiento de los individuos creciendo en su hábitat natural, además de determinar las relaciones que tiene el crecimiento de la especie en Valdivia con la variabilidad climática local. La hipótesis principal sostiene que, las tasas de crecimiento anual en Valdivia son mayores que los que registra la especie en su hábitat natural, dada la mayor precipitación anual y temperaturas más moderadas, con una estación seca menos marcada que en su hábitat natural.

\section{MÉTODOS}

Área de estudio. La plantación de A. pinsapo estudiada se encuentra en el Arboreto de la Universidad Austral de Chile, en adelante arboreto, ubicado en la Isla Teja (39 $48^{\prime}$ '06" $\mathrm{S} ; 7^{\circ} 15^{\prime} 40^{\prime \prime} \mathrm{O}$ ) dentro de la ciudad de Valdivia, en la región de Los Ríos, Chile (figura 1).

La pluviometría de Valdivia es considerablemente elevada, pudiendo llegar a acumularse más de $3000 \mathrm{~mm}$ en años especialmente lluviosos, aunque la media anual durante el periodo 1901-2005 fue de 2.442,5 mm (GonzálezReyes y Muñoz 2013). En este lugar, las precipitaciones presentan un descenso en primavera y verano, sin lograr condiciones de sequía estival (figura 1). La temperatura es moderada, con una media de $12,4{ }^{\circ} \mathrm{C}$ y una amplitud térmica anual de $8,8^{\circ} \mathrm{C}$, con un número muy bajo de heladas en el año (Huber 1970).

El arboreto se encuentra en la ecorregión denominada "bosque valdiviano", que abarca la zona centro-sur de Chile y algunas zonas fronterizas del sudoeste de Argentina. Se caracteriza por un clima oceánico, templado-lluvioso, con la peculiaridad de ser el único de estas características en América del Sur (Luebert y Pliscoff 2005). La Ecorregión Valdiviana se extiende desde el paralelo $33^{\circ} \mathrm{S}$ hasta el paralelo $46^{\circ} \mathrm{S}$, abarcando una superficie de más de $300.000 \mathrm{~km}^{2}$ limitados al este por la Cordillera de Los Andes y al oeste y sur por el océano Pacífico (Luebert y 


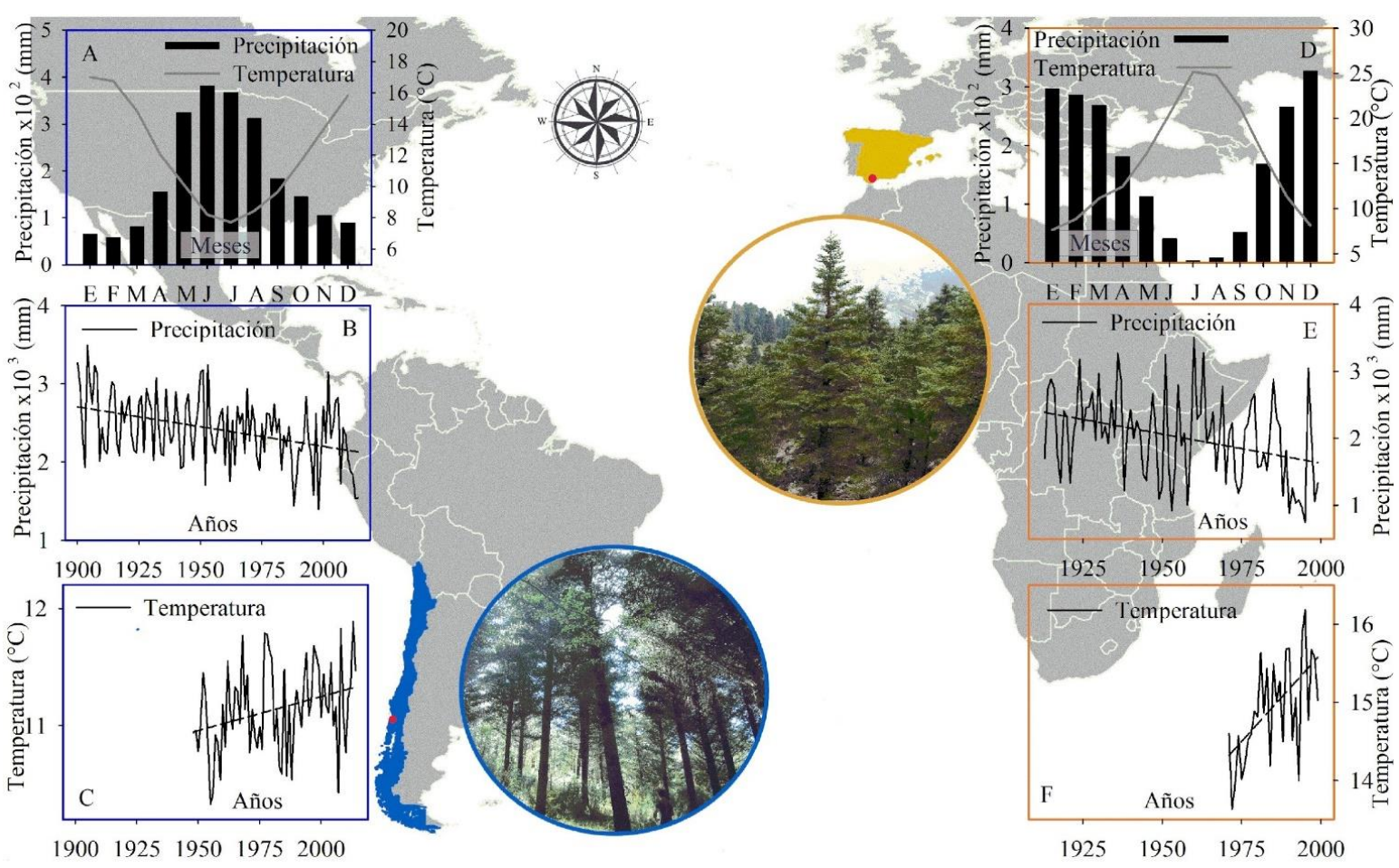

Figura 1. Ubicación geográfica de la plantación de A. pinsapo en Valdivia (Chile) y de las poblaciones naturales de la especie en Grazalema (España). A) Climograma de Valdivia. B) Tendencias de las precipitaciones en Valdivia. C) Tendencia en temperatura de Valdivia. D) Climograma de Grazalema. E) Tendencias de las precipitaciones en Grazalema. F) Tendencia de la temperatura en Grazalema.

Geographical location of the A. pinsapo plantation in Chile and of the natural stands of the species in Spain. A) Climograph of Valdivia. B) Precipitation tendencies in Valdivia. C) Temperature tendencies in Valdivia. D) Climograph of Spain. E) Precipitation tendencies in Spain. F) Temperature tendencies in Spain.

Pliscoff 2005). Los registros meteorológicos de los últimos 150 años evidencian un descenso importante de las precipitaciones anuales en la ciudad de Valdivia (figura 1), así como una extensión de la estación estival, explicada por otoños más secos y una concentración de las precipitaciones en invierno, lo cual ha sido atribuido en parte, a la influencia de forzantes climáticos globales que afectan a la región, como el Modo Anular del Sur (SAM; GonzálezReyes y Muñoz 2013).

Los individuos muestreados en la plantación del arboreto están situados a unos $46 \mathrm{~m}$ sobre el nivel del mar con una pendiente media del $22 \%$ y una exposición suroeste (cuadro 1), por lo que se encuentra en condiciones de umbría o semiumbría. En cuanto al sotobosque, los pinsapos se encontraban asociados principalmente con helechos, hiedras y otras herbáceas, además de arrayanes (Luma apiculata DC. Burret.) de pequeño tamaño. La densidad de la plantación se determinó a través de un censo en un área de $646,25 \mathrm{~m}^{2}$, donde se contaron 28 ejemplares, resultando una densidad de 433 árboles por hectárea.

El rodal de A. pinsapo fue plantado en 1976 con ejemplares de 5 años de edad ( 2 años en almaciguera y posteriormente trasplantados a raíz desnuda en platabandas por 3 años), provenientes de la Yunquera, Málaga en España.
Para comparar los crecimientos de los pinsapos del sitio de Valdivia (Chile) con ejemplares en su hábitat natural (España), se utilizaron datos de los sitios Nieves II y Pinar (Génova y Muñoz 2005, Génova 2007), que se ubican en la sierra de las Nieves en la provincia de Málaga, y en la sierra de Grazalema en la provincia de Cádiz, respectivamente.

Características de estructura y crecimiento del rodal de Abies pinsapo en Valdivia. Para estimar la altura media del rodal de Valdivia, se utilizó un hipsómetro Suunto para tomar dos lecturas a 9 de 18 individuos seleccionados de la plantación para la obtención de muestras dendrocronológicas. Los árboles seleccionados fueron aquellos que presentaban una mejor visibilidad de la copa y de su altura total. Los diámetros de los mismos árboles se midieron utilizando una cinta diamétrica a una altura de 1,30 m desde el nivel del suelo.

Para analizar el crecimiento anual de los árboles, se seleccionaron todos aquellos individuos que no se encontraban creciendo en el borde de la plantación, a los cuales se extrajo dos tarugos o testigos de madera (perpendiculares entre sí) de cada árbol a 0,3 m desde el suelo mediante barrenas de Pressler, procurando que estos llegaran al 
Cuadro 1. Caracterización de las formaciones de Abies pinsapo estudiadas, y de las series de crecimiento analizadas luego del procedimiento de datación cruzada (Fuente sitios Nieves II/ Pinar: Génova 2007).

Characterization of Abies pinsapo formations studied, and of the growth series analyzed (Source for Nieves II/Pinar sites: Génova 2007).

\begin{tabular}{lccc}
\hline & Nieves II & Pinar & Valdivia \\
\hline Municipio & Yunquera & Grazalema & Valdivia \\
Provincia/Región & Málaga & Cádiz & Los Ríos \\
País & España & España & Chile \\
Latitud & $36^{\circ} 41^{\prime} \mathrm{N}$ & $36^{\circ} 46^{\prime} \mathrm{N}$ & $39^{\circ} 48^{\prime} \mathrm{S}$ \\
Longitud & $4^{\circ} 58^{\prime} \mathrm{W}$ & $5^{\circ} 25^{\prime} \mathrm{W}$ & $73^{\circ} 15^{\prime} \mathrm{O}$ \\
Altitud (msnm) & $1050-1250$ & $1150-1350$ & 46 \\
Orientación & $\mathrm{NE}$ & $\mathrm{N}$ & $\mathrm{SO}$ \\
Pendiente media (\%) & 30 & 50 & 22 \\
$\mathrm{~N}^{\circ}$ árboles/ $\mathrm{N}^{\circ}$ series & $14 / 25$ & $19 / 32$ & $32 / 54$ \\
$\mathrm{~N}^{\circ}$ de anillos máximo, medio y mínimo & $106,82,53$ & $214,97,40$ & $36,31,22$ \\
Ancho de anillo medio (mm) y desviación estándar & $2,70 \pm 1,65$ & $2,06 \pm 1,33$ & $3,80 \pm 1,45$ \\
Ancho de anillo medio (mm) y desviación estándar primeros 36 años & $4,09 \pm 1,96$ & $3,56 \pm 2,29$ & $3,80 \pm 1,45$ \\
\hline
\end{tabular}

centro del fuste. De esta manera, se barrenaron 18 árboles remanentes de la plantación original, obteniendo 36 testigos de madera. Paralelamente, se analizaron 14 rodelas de todos los ejemplares raleados en el año 2007, las que también formaron parte del material estudiado. Transcurrido el tiempo de secado, el material leñoso se pulió en el plano transversal del leño con lijadora orbital comenzando con lijas de granulometría gruesa (100 granos $\left.\mathrm{cm}^{-2}\right)$ hasta terminar con lijas de grano más fino $\left(1.000 \mathrm{~g} \mathrm{~cm}^{-2}\right)$. Esto, con el fin de distinguir visualmente los límites de los anillos. El ancho de los anillos de todas las muestras fue medido con WinDendro (Regents Instruments) para lo cual, se escanearon previamente todas las muestras con un escáner Epson Expression 11000XL, Versión Itrax a 1200 DPI, calibrado para reconocer distancias en las imágenes utilizando este software. A las rodelas se les midieron dos radios en dirección perpendicular, obteniendo dos series de ancho de anillo por cada rodela.

Para corroborar el fechado visual de las muestras se utilizó COFECHA (Holmes 1983), que permite corregir errores en la datación comparando los patrones de crecimiento entre muestras, utilizando segmentos traslapados. A este procedimiento se le conoce como datación cruzada o cofechado (Fritts 1976). Posteriormente, para construir la cronología de ancho de anillos, se seleccionaron solamente aquellas muestras que presentaron valores estadísticos elevados de intercorrelación entre series $(r>0,5)$. Dado que las muestras fueron recogidas en septiembre de 2015 y la última estación de crecimiento en el hemisferio sur comenzó en la primavera de 2014, el último anillo formado (el más próximo a la corteza) fue datado como 2014 siguiendo la convención de Schulman (1956). Utilizando estas series de crecimiento sin estandarizar, se calcularon el incremento corriente anual (ICA) y el incremento medio anual (IMA). El ICA corresponde al crecimiento radial producido cada año por los árboles de la plantación, mientras que el IMA es la media del crecimiento total a cierta edad de un árbol. Ambos conceptos son ampliamente utilizados en el ámbito forestal, principalmente para determinar la edad óptima de rotación o corta de una plantación y maximizar el volumen (ICA = IMA). Calcular ambos incrementos tuvo como objetivo determinar los patrones del crecimiento radial de la masa forestal en función de la edad, logrando también evaluar la respuesta del crecimiento radial de los individuos de la plantación posterior al raleo del año 2007. Se utilizó el software "InfoStat" para aplicar un ajuste de media móvil o "suavizado", de amplitud 5 años, a la curva del ICA de la plantación con el fin de hacer más explícito el patrón medio de crecimiento de la especie en Valdivia.

Para evaluar un posible efecto diferencial de la variabilidad climática en distintas estaciones del año, sobre el crecimiento de $A$. pinsapo en Valdivia, se incluyeron en este estudio mediciones dendrocronológicas del ancho total de los anillos de crecimiento, además de la diferenciación entre madera temprana y tardía.

Caracterización de las masas españolas de Abies pinsapo empleadas en este estudio. El sitio Nieves II se encuentra ubicado en el Parque Natural de la sierra de las Nieves, en la provincia de Málaga (cuadro 1, figura 1). Corresponde a un tipo de bosque de relativa homogeneidad en cuanto a densidad, con crecimientos vigorosos, alcanzando diámetros normalmente superiores a $100 \mathrm{~cm}$ para una media de edades inferior a 100 años. El sitio Pinar, ubicado en 
la sierra el Pinar, a su vez perteneciente al Parque Natural sierra de Grazalema (entre las provincias de Cádiz y Málaga), está compuesto por masas aisladas y de apariencia longeva, que subsisten en suelos esqueléticos y elevadas pendientes en torno a una masa principal de individuos más jóvenes (Génova 2007).

Estos sitios se ubican en la zona de clima mediterráneo, caracterizado por una concentración de las precipitaciones en los meses de otoño e invierno, y una sequía que se extiende desde el final de la primavera y a lo largo de todo el verano (Génova 2007, figura 1). No obstante, se trata de un clima mediterráneo poco común, pues registra precipitaciones en torno a $2.000 \mathrm{~mm}$ anuales en la sierra de Grazalema (con valores máximos de hasta $4.300 \mathrm{~mm}$ anuales) y por encima de los $1.000 \mathrm{~mm}$ en la sierra de las Nieves (Gómez-Zotano et al. 2015).

A pesar de su reducida distribución y la amenaza constante a causa de incendios forestales y el cambio climático, las masas de los sitios de estudio han experimentado una expansión notable en los últimos 80 años, pasando de 200 a 418 ha en la sierra del Pinar y de 1.000 a 2.871 ha en la Sierra de Las Nieves en el período entre 1933 y 2008 (Esteban et al. 2010).

Datos de crecimiento de Abies pinsapo en España y en Valdivia. Se utilizaron datos no estandarizados de los sitios Pinar y Nieves II de pinsapos en España, seleccionando de cada sitio los diez individuos que mostraron mayor crecimiento promedio en sus primeros 43 años para poder realizar una comparación directa con los 10 individuos de mayor crecimiento en el sitio de Valdivia, considerando solamente los años biológicos de los árboles. Posteriormente, se compararon gráficamente las series de crecimiento medio de los tres sitios a lo largo de sus primeros 43 años de vida (figura 3).

Construcción de cronologías y relación clima-crecimiento de Abies pinsapo en Valdivia. Mediante el programa WinDendro (Regent Instruments) se midió el ancho total de los anillos (RW, por sus siglas en inglés) de cada serie de crecimiento, así como el ancho correspondiente a la madera temprana (EW, por sus siglas en inglés) y a la madera tardía (LW, por sus siglas en inglés). La madera temprana se forma durante los primeros meses del período vegetativo del árbol, hasta que la reducción del fotoperiodo o el descenso de la disponibilidad hídrica inducen la producción de traqueidas más pequeñas y aplanadas, con paredes celulares más anchas y lignificadas y lúmenes más estrechos, dando paso a la formación de la madera tardía (Uggla et al. 2001). Esto hace que ambas partes sean diferenciables visualmente en un microscopio, debido a su coloración más clara en la madera temprana y obscura en la tardía. El estudio de la madera temprana y tardía permite profundizar en la ecología de las especies a escala temporal intra-anual, explorando los factores y condiciones que controlan el crecimiento en distintos momentos dentro de su período vegetativo (Pompa-García y Camarero-Martínez 2015). En el caso de los pinsapos analizados en este estudio (ejemplares chilenos) el límite entre madera temprana y madera tardía fue fácilmente reconocible por su marcada diferencia de coloración.

La elaboración de las tres cronologías de crecimiento de la plantación se llevó a cabo con el software ARSTAN (Cook 1985). Este software permite estandarizar las series de ancho de anillos eliminando la variabilidad del crecimiento inducida por factores individuales de cada árbol, y también la autocorrelación de primer orden dentro de las series de crecimiento. De esta manera, con ARSTAN es posible escoger la curva (función matemática) que mejor se ajuste al crecimiento de cada una de las series para eliminar las tendencias del crecimiento inducidas por perturbaciones endógenas del rodal, que no son directamente atribuibles al clima, como es la edad del árbol o la competencia, maximizando así la señal climática. Por esto, se utilizó una curva exponencial negativa o una línea recta para estandarizar las series de crecimiento de la plantación de Valdivia.

Posterior al proceso de estandarización, las series fueron promediadas en curvas que representan el crecimiento medio de la población de árboles muestreados (media aritmética), donde las series de crecimiento muestran una media cercana a uno y varianza homogénea. Estos promedios son llamados cronologías de índice de ancho de anillo. La calidad de estas cronologías fue controlada a través de un estadístico llamado Señal Expresada de la Población (EPS, por sus siglas en inglés). El EPS analiza la calidad estadística de la cronología media a través de la comparación con una cronología hipotética infinitamente replicada (Wigley et al. 1984). Se seleccionaron periodos donde el EPS superó el umbral de 0,85 para comparar el crecimiento de los individuos de la plantación con la variabilidad climática local.

Se utilizaron datos de precipitación acumulada y temperatura media de la estación de Isla Teja, en Valdivia para representar el clima local. Utilizando funciones de correlación (Blasing et al. 1984), se buscaron las mayores correlaciones de Pearson entre las cronologías estándar y residual de las tres series de crecimiento (RW, EW y LW) con todas las combinaciones posibles en un periodo de 36 meses de precipitación acumulada y temperatura media de Valdivia para cada año de cada cronología (figura 4). Estas funciones permiten analizar las relaciones del crecimiento con el clima, considerando periodos climáticos dentro de la estación de crecimiento y hasta dos años previos a la formación del anillo.

\section{RESULTADOS}

Crecimiento del rodal de Abies pinsapo en Valdivia. El rodal presentó una densidad de 434 árboles por hectárea, un DAP medio de $30,27 \mathrm{~cm}$ y una altura media cercana a 19 $\mathrm{m}$ (cuadro 2). 
Cuadro 2. Características del rodal de A. pinsapo en Valdivia (Chile) y de los sitios Nieves II y Pinar en España, junto con los correspondientes estadísticos de la cronología de ancho de anillo de la especie en cada sitio. SM = Sensibilidad media.

Characteristics of the A. pinsapo stand in Valdivia (Chile) and Nieves II and Pinar sites in Spain, together with their corresponding statistics for the tree-ring width chronologies of the species in each site. $\mathrm{SM}=$ Mean sensitivity.

\begin{tabular}{lccccccc}
\hline \multicolumn{1}{c}{ Sitio } & $\begin{array}{c}\text { DAP } \\
(\mathrm{cm})\end{array}$ & $\begin{array}{c}\text { Altura } \\
(\mathrm{m})\end{array}$ & $\begin{array}{c}\text { Densidad } \\
(\text { árboles/ha) }\end{array}$ & $\begin{array}{c}\text { Periodo } \\
(\mathrm{años})\end{array}$ & SM & $\begin{array}{c}\text { Inter-correlación } \\
(P<0,05)\end{array}$ & $\begin{array}{c}\text { Auto- correlación } \\
(P<0,05)\end{array}$ \\
\hline Valdivia & 30,27 & 18,83 & 434 & $1972-2014$ & 0,25 & $\mathrm{r}=0,66$ & $\mathrm{r}=0,69$ \\
NievesII & 112 & - & - & $1887-1998$ & 0,23 & $\mathrm{r}=0,56$ & $\mathrm{r}=0,84$ \\
Pinar & 91 & - & - & $1788-1999$ & 0,31 & $\mathrm{r}=0,48$ & $\mathrm{r}=0,77$ \\
\hline
\end{tabular}

De los 32 árboles medidos en Valdivia, se obtuvieron 54 series que presentaron buenos estadísticos de datación cruzada, logrando una intercorrelación entre series de $\mathrm{r}=$ $0,66(P<0,05)$, una sensibilidad media de 0,25 y una autocorrelación de primer orden de 0,69 (cuadro 2). La sensibilidad media es una medida de la diferencia en el ancho del anillo del árbol y varía de un año a otro entre 0 a 1 . La intercorrelación es un índice de similitud que va de -1 a 1 y compara el ancho de los anillos de cada serie de crecimiento según su año calendario en segmentos de largo variable, siendo el largo estándar de 50 años con traslape de 25 años entre segmentos. Mientras el segmento sea más pequeño, la rigurosidad de la intercorrelación es mayor y se pueden detectar errores de fechado con mayor facilidad en series cortas. Por su parte, la autocorrelación de primer orden se basa en la comparación del crecimiento de un año con respecto al año anterior, y permite evaluar cuanto influye el crecimiento de un año a otro (Fritts 1976).

El análisis conjunto de las funciones de incremento medio anual (IMA), incremento corriente anual (ICA) y crecimiento acumulado, permitió observar el ritmo de crecimiento radial de la masa a lo largo de los años de vida de los árboles (figura 2). Las curvas se analizaron utilizando datos no estandarizados, permitiendo evaluar los cambios de ritmo de crecimiento radial causados por diversos factores no particularmente climáticos, como la edad de los árboles o la competencia entre individuos. El ajuste de media móvil (5 años) permitió apreciar que, en el momento previo al raleo del año 2007, las funciones ICA e IMA estaban próximas a interceptarse como resultado de una desaceleración del crecimiento años previos a la intervención, atribuible a la competencia entre individuos y al cierre de copas dentro de la plantación.

Comparación del crecimiento de Abies pinsapo en Valdivia (Chile) y en los sitios de España. El promedio de crecimiento durante los 43 años de las series seleccionadas de Valdivia fue de 4,44 $\mathrm{mm}$ anuales. La plantación alcanzó un máximo de crecimiento entre los años 1996 y 2000 fluctuando entre 5,3 y $5,6 \mathrm{~mm}$, respectivamente. Por su parte, los crecimientos menores fluctuaron entre 0,65 y $0,55 \mathrm{~mm}$ entre los años 1975 y 1977, respectivamente (figura 2).

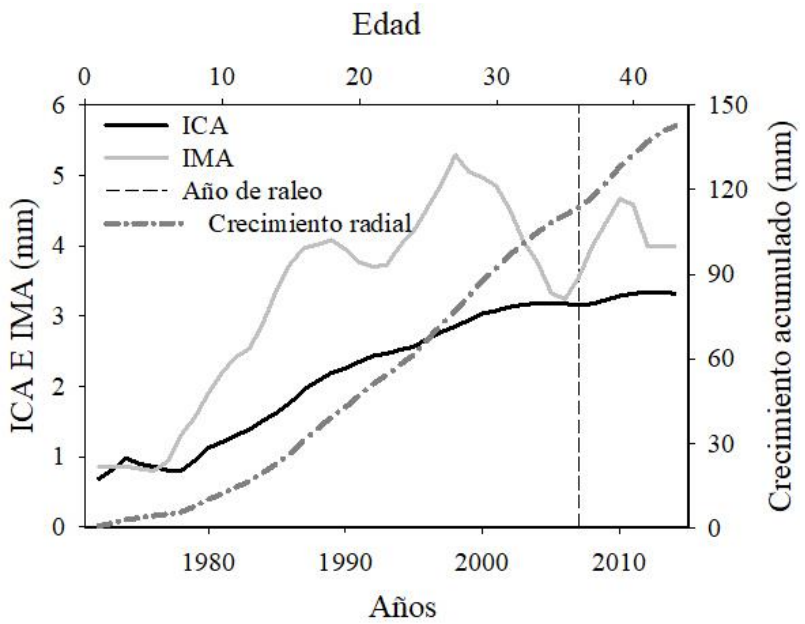

Figura 2. Funciones de crecimiento acumulado, IMA e ICA, de la plantación de Abies pinsapo en Valdivia. El ICA se muestra ajustado a través de una media móvil de cinco años. La línea vertical discontinua señala el año en que se produjo el raleo.

Cumulative growth functions, IMA (Mean Annual Increment) and ICA (Periodic Annual Increment) for the Abies pinsapo plantation in Valdivia. The ICA has been adjusted using a five-year moving average. The discontinuous vertical line indicates the year of thinning.

Estos árboles presentaron un ritmo de crecimiento lento en sus primeros 10 años de vida, inferior a $2 \mathrm{~mm}$ anuales, que gradualmente se aceleró durante los siguientes 20 años para alcanzar los máximos de crecimiento a la edad de 30 años. Posteriormente, sus crecimientos volvieron a disminuir los siguientes siete años, después del cual respondieron positivamente al raleo a la edad de 37 años. Posteriormente disminuyen las tasas de crecimiento $c a .4 \mathrm{~mm} / \mathrm{año} \mathrm{a}$ la edad de 43 años (figura 3). La liberación de competencia a causa del raleo permitió retomar súbitamente las tasas de crecimiento previas a la intervención por un corto período de tiempo, ya que 3 años después los crecimientos volvieron a disminuir (figura 3 ).

Se observó un patrón distinto en las 20 series seleccionadas de A. pinsapo de los dos sitios de España, cuyo crecimiento comenzó siendo relativamente elevado con 


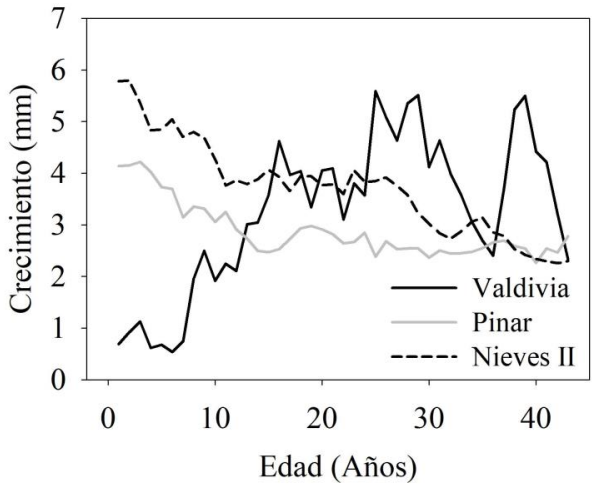

Figura 3. Comparación de los crecimientos radiales medios anuales de series seleccionadas de los sitios de Valdivia, Pinar y Nieves II en sus primeros 43 años de desarrollo.

Comparison of the mean annual radial growth of selected series of Valdivia, Pinar and Nieves II sites in their first 43 years of development. valores cercanos a $6 \mathrm{~mm}$ anuales en los primeros 10 años, y luego disminuye gradualmente hasta alrededor de $4 \mathrm{~mm}$ anuales a la edad de 40 años. En general, se observó que el promedio de crecimiento de las series de Valdivia fue inferior en los primeros 20 años y superior en la segunda mitad de la edad de los árboles de los sitios de España (figura 3).

Relación clima-crecimiento en el rodal de Abies pinsapo de Valdivia. Los resultados obtenidos a partir de funciones de correlación para las tres cronologías (RW, EW y LW), presentaron correlaciones positivas con el promedio de la temperatura y negativas con la suma de las precipitaciones en los veranos previos a la estación de crecimiento (septiembre-marzo). Por su parte, correlaciones positivas con la precipitación invernal del año corriente y previo también fueron observadas con cronologías estándar y residual de la especie (figura 4). Las correlaciones alcanzadas

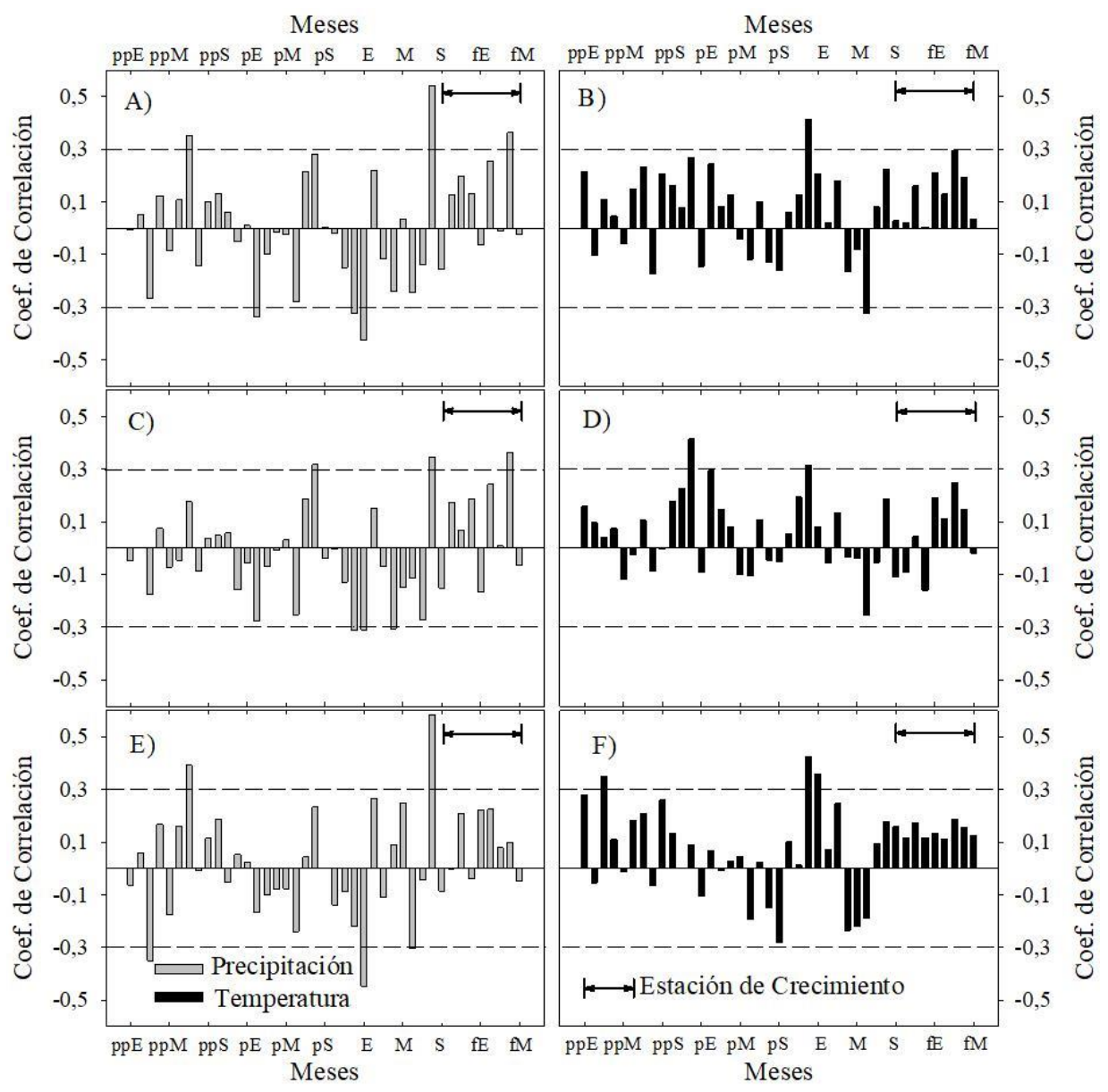

Figura 4. Función de correlación del crecimiento de Abies pinsapo en Valdivia, considerando la estación de crecimiento en la cual se formó el anillo, uno (p) y hasta dos años previos (pp). A) Ancho total de anillo y precipitación. B) Ancho total de anillo y temperatura. C) Madera temprana y precipitación. D) Madera temprana y temperatura. E) Madera tardía y precipitación. F) Madera tardía y temperatura. Todas las correlaciones fueron calculadas para un nivel de significancia del $95 \%(P<0,05)$.

Response function of growth of Abies pinsapo in Valdivia, considering the growing season in which the ring was formed, and two years before current growth. A) Total ring width and precipitation. B) Total ring width and mean temperature. C) Early wood and precipitation. D) Early wood and mean temperature. E) Late wood and precipitation. F) Late wood and mean temperature. All correlations were calculated with a significance level of $95 \%(P<0.05)$. 
entre las variables climáticas y el ancho total RW, por un lado, y entre estas mismas variables y la madera tardía LW, por el otro, fueron más similares entre sí que las correlaciones entre clima (precipitación, temperatura) y madera temprana EW y clima y ancho total RW (figura 4).

La cronología de ancho total en su versión residual (RW) presentó altas correlaciones con la precipitación de agosto: $\mathrm{r}=0,52, P<0,05$ (figura $4 \mathrm{~A}$ ), y con la temperatura de diciembre del año previo: $\mathrm{r}=0,50, P<0,05$ (figura 4B). En cuanto a la madera temprana EW, las correlaciones con la precipitación fueron positivas y marginalmente significativas con meses específicos como agosto y febrero del año corriente de formación del anillo, pero negativas y marginalmente significativas con meses como diciembre y enero del año previo a la formación del anillo (figura 4C). También la cronología EW en su versión estándar presentó correlaciones positivas con la temperatura de diciembre de dos años previos (ppD) (figura 4D). Por su parte, la cronología de madera tardía LW en su versión residual correlacionó con la precipitación de agosto principalmente (figura 4E) $\mathrm{y}$ con la temperatura de diciembre y enero (pD-J) del año previo (figura 4F).

Las relaciones clima-crecimiento (figura 5) muestran que la precipitación de agosto (figura 5A), justo antes del

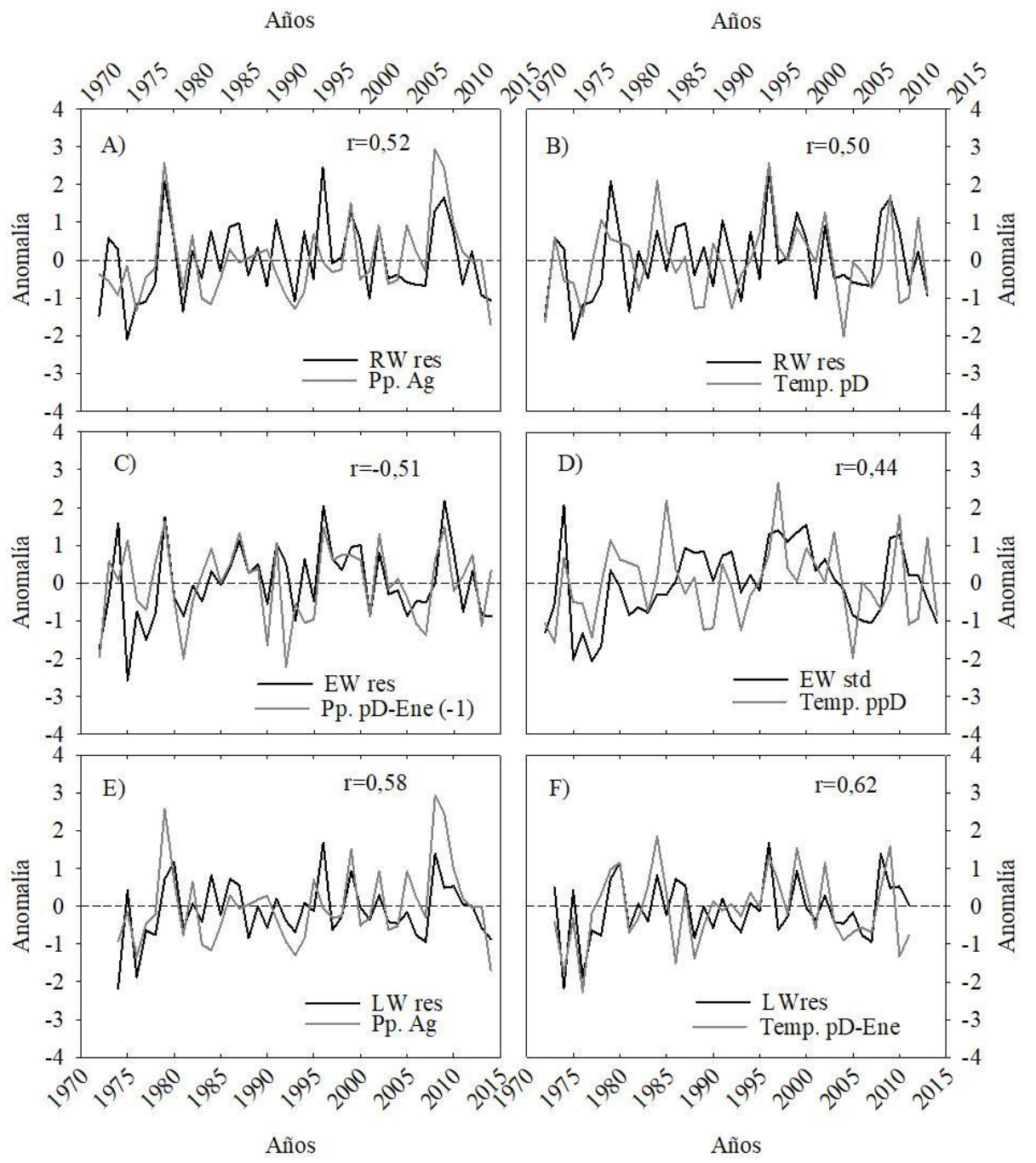

Figura 5. Relaciones crecimiento-clima de Abies pinsapo en Valdivia. A) Ancho total de anillo y precipitación del mes de agosto corriente. B) Ancho total de anillo y temperatura del mes de diciembre del año previo. C) Madera temprana y precipitación de los meses de diciembre y enero del año previo. D) Madera temprana y temperatura de diciembre de dos años previos. E) Madera tardía y precipitación de agosto corriente. F) Madera tardía y temperatura de los meses de diciembre y enero del año previo. Todas las correlaciones fueron calculadas para un nivel de significancia del $95 \%(P<0,05)$.

Growth-climate relationships of Abies pinsapo in Valdivia. A) Total ring width and current August precipitation. B) Total ring width and previous year's December mean temperature. C) Early wood and previous year's December and January precipitation. D) Early wood and mean temperature of December two years before growth. E) Late wood and current August precipitation. F) Late wood and previous year's December and January mean temperature. All correlations were calculated with a significance level of $95 \%(P<0.05)$. 
inicio de la estación de crecimiento en Valdivia, y la temperatura de diciembre del año previo (figura 5B), controlan el crecimiento del anillo en su ancho total RW. Sin embargo, la madera temprana EW se correlacionó negativamente con la precipitación de los meses de diciembre y enero del año previo a la formación del anillo (figura 5C), mientras que con la temperatura tuvo una relación positiva en el mes de diciembre de dos años previos (figura 5D). Por su parte, la madera tardía LW presentó la mayor correlación con la precipitación de agosto del año corriente (figura 5E) de las tres cronologías de ancho de anillo (RW, EW y LW). También la correlación que alcanzó esta cronología con la temperatura de los meses de verano del año previo a la formación del anillo fue la más alta de las tres analizadas de la plantación de Valdivia (figura 5F).

\section{DISCUSIÓN}

Comparación de los crecimientos entre Abies pinsapo de Chile y España. Entre los ejemplares de Valdivia y los que se desarrollaron en los sitios Nieves II y Pinar se observaron tendencias opuestas de crecimiento. Mientras que en los sitios españoles los abetos se desarrollaron con rápidos crecimientos durante sus primeros años, en Valdivia lo hicieron a un ritmo inicial mucho menor. No obstante, la tendencia del crecimiento radial de los primeros 40 años en la plantación de Valdivia se mantuvo al alza mientras que en los sitios de España el crecimiento de los abetos fue decreciendo con el tiempo, generando una tendencia negativa en la serie de crecimiento promedio durante las primeras cuatro décadas de su establecimiento. Esto se puede explicar por las diferentes condiciones ecológicas y de competencia en cada caso. Mientras en Chile, los pinsapos se establecieron en un sitio abierto con un distanciamiento homogéneo, en España los pinsapos en condiciones naturales podrían presentar más competencia por recursos desde el inicio de su establecimiento.

En el caso de los pinsapos de Valdivia, estos sufrieron importantes limitaciones durante sus primeros años de vida a consecuencia del manejo de las plantas en su etapa de viverización. En primer lugar, fueron expuestas a fuertes restricciones para el desarrollo de su sistema de raíces, aspecto muy importante para el desarrollo de esta especie (Soto et al. 2001). Posteriormente se agregó el estrés provocado por el traslado y trasplante al arboreto, lo que supuso una fase de adaptación a las nuevas condiciones. Esto se ve reflejado en sus bajos crecimientos en los primeros 5 a 10 años de vida (figura 3). Tras la etapa de establecimiento, los crecimientos anuales fueron en aumento, hasta igualar y finalmente sobrepasar las tasas de crecimiento de los sitios Nieves II y Pinar.

Nieves II y Pinar presentaron tendencias de crecimiento muy similares, decrecientes desde los primeros años, en ambos casos posiblemente debido al aumento de la competencia. Cerca de los treinta años de edad, el sitio Pinar sufrió un nuevo decaimiento, posiblemente por las limita- ciones que los suelos esqueléticos del lugar imponen al desarrollo más allá del tamaño alcanzado a esta edad. El crecimiento de los individuos también hace que se equiparen al resto de la masa y que por tanto se vean más expuestos a las inclemencias de zonas montañosas al desarrollarse. Estas restricciones no se presentan en la plantación de pinsapos de Valdivia, con árboles espaciados y equidistantes entre ellos, pendientes suaves, suelos profundos y de estructura óptima, moderadamente ácidos, además de contar con valores elevados de carbono y nitrógeno, según estudios desarrollados en áreas próximas a la de la plantación estudiada dentro del arboreto (Navarrete 2006).

Tras un máximo de crecimiento radial cercano a los $8 \mathrm{~mm}$ anuales, la plantación de Valdivia registró una disminución en el crecimiento, lo cual es atribuido al cierre de copas, y probablemente también a la competencia radicular, la cual según Soto et al. (2001) se manifiesta con anterioridad a la tangencia de copas, causando incluso un mayor perjuicio sobre el desarrollo del rodal. Posteriormente se observó un rápido repunte tras el raleo ocurrido en el año 2007, que alcanzó hasta los $7 \mathrm{~mm}$ anuales y que se mantuvo durante dos años para nuevamente disminuir notablemente en años posteriores. Estas circunstancias podrían estar relacionadas con las condiciones de sequía que se han dado durante el último periodo en Valdivia. Aunque también podría significar que el raleo llevado a cabo fue insuficiente para recuperar las mayores tasas de crecimiento presentadas por los árboles de esta plantación previo a la intervención. En este sentido es posible que una menor densidad de árboles resulte en tasas de crecimiento aún mayores. Posiblemente, un segundo raleo luego de dos a tres años desde la primera intervención podría haber sido oportuno para mantener por un periodo más prolongado las tasas de crecimiento que alcanzó tras la primera intervención, que si bien no fueron las mayores, mejoraron sustancialmente su desarrollo en diámetro.

Relación clima-crecimiento en Abies pinsapo en Valdivia, Chile. A partir de los resultados obtenidos en la plantación de $A$. pinsapo en Valdivia, se puede deducir que su crecimiento está controlado principalmente por dos factores climáticos. El primero está asociado a las condiciones del clima de verano, donde el crecimiento es favorecido por un aumento de temperatura con escasa precipitación en los primeros meses del verano del año previo a la formación del anillo. Por otro lado, el segundo factor climático que favorece el crecimiento es un buen aporte hídrico en el invierno que precede a la estación de crecimiento, la cual comienza en los meses de primavera en estas latitudes. Estos factores no son los mismos que en su hábitat original, donde el pinsapo responde positivamente a la precipitación y negativamente a la temperatura de los últimos meses del verano previo al periodo de crecimiento, $\mathrm{y}$ positivamente también a la precipitación de la primavera e inicio del verano actual (Génova 2007). Tanto en España como en Chile, el crecimiento de la especie tiene influen- 
cia significativa de las variables climáticas del año previo al crecimiento, lo cual parece depender de las reservas de carbono almacenadas de un año a otro (van der Maaten 2012). En el caso de España, esto también se ha vinculado en cierta medida con el agua almacenada en el suelo (Génova y Muñoz 2005).

En España, el verano es un periodo crítico para el crecimiento arbóreo dada las altas temperaturas y escasa precipitación. En Valdivia, la diferencia de precipitación entre las distintas estaciones del año es marcada pero no existe un periodo crítico de sequía, alcanzando el promedio de precipitación de febrero, el mes más seco, valores $c a .63$ $\mathrm{mm}$ (período 1900-2005). Las temperaturas se mantienen moderadas en las distintas estaciones del año, con una temperatura media anual de $12,2^{\circ} \mathrm{C}$ y una temperatura media para enero (mes más cálido) de $17,0^{\circ} \mathrm{C}$ (período 19612005), lo que las asemeja a las cotas superiores de los macizos de las Nieves y Grazalema (Gómez-Zotano et al. 2015). Este clima favorece un inicio vegetativo anticipado con respecto al clima español, gracias a las suaves temperaturas primaverales y la disponibilidad de agua posterior al invierno, a partir de las reservas almacenadas en años anteriores, y que coincide con el desarrollo de la madera temprana. Estas reservas se pueden acumular gracias a condiciones de escasa precipitación y temperaturas elevadas durante periodos estivales previos. Posteriormente se forma la madera tardía, que depende en mayor medida de la precipitación invernal del año corriente, principalmente del mes de agosto, último mes del invierno y cuya pluviosidad en cierta manera marca la disponibilidad de agua en los meses venideros que dan inicio al periodo vegetativo.

De acuerdo con los análisis de correlación efectuados en este trabajo, parece correcto argumentar que la temperatura constituye el principal factor limitante para el crecimiento de la especie en Valdivia, ya que es capaz de explicar más del $36 \%$ de la variabilidad en el crecimiento tanto anual como de la madera temprana y tardía. Este factor está fuertemente asociado a la insolación en estas latitudes, lo cual resulta restrictivo para el crecimiento pues la región de estudio se encuentra en el límite inferior en cuanto a insolación necesaria para la especie, siendo este, según Soto et al. (2001), de 1.700 horas de sol al año. La ciudad de Valdivia recibe una insolación media de 1.720 horas al año, por lo que años con abundante precipitación y nubosidad pueden limitar su crecimiento. Esto no sucede así con los sitios Nieves II y Pinar, cuyas correlaciones con el clima son algo más bajas con las variables precipitación y temperatura de la zona, indicando que la mayor parte de la varianza del crecimiento podría estar explicada por otros factores como pueden ser condiciones locales, influencia antrópica, entre otros (Génova y Muñoz 2005).

Los actuales cambios en el clima de Valdivia, caracterizados por una extensión de la estación seca estival y concentración de las lluvias en invierno, así como una marcada reducción de las precipitaciones (González y Muñoz 2013), que es también una característica del clima futuro proyectado para esta zona (CONAMA 2006), podrían significar condiciones aún más favorables para este abeto en la zona, pues la precipitación sería todavía suficiente para sostener su crecimiento, y la insolación aumentaría. Las condiciones limitantes para el crecimiento de la especie en Valdivia ocurren mayormente en veranos de bajas temperaturas y elevadas precipitaciones. En resumen, a partir de estos resultados en Chile y de estudios previos acerca de la ecología de esta especie en España, es posible que $A$. pinsapo vea limitado su crecimiento por condiciones de baja luminosidad, como las creadas por veranos de abundante nubosidad (precipitación elevada, baja temperatura), no siendo la humedad un factor limitante.

Ocupación potencial de la especie fuera de su distribución natural. Abies pinsapo es una especie catalogada "en peligro" dentro de las categorías de conservación de la UICN (Linares y Carreira 2006), debido a la reducida extensión que ocupa y la gran presión que se cierne sobre sus poblaciones, tanto histórica como actual (Esteban et al. 2010). La información obtenida de la plantación de Valdivia, así como de otros ensayos llevados a cabo en España (Soto et al. 2001), dejan abierta la puerta a una expansión de la especie en zonas no habituales y no necesariamente similares en cuanto a condiciones edáficas y climáticas respecto de su hábitat natural, corroborando el potencial de la especie para ser conservada ex-situ.

Esta expansión en su hábitat natural ya se viene produciendo durante los últimos 80 años como se mencionó anteriormente. Esto indica que, si bien su distribución actual es fruto de la orogénesis alpina y las glaciaciones cuaternarias (Soto et al. 2001), la deforestación, el sobrepastoreo y los incendios son los responsables de que este abeto solo habite enclaves aislados y de difícil acceso (Esteban et al. 2010). Por lo tanto, cabe pensar que es altamente probable que el control y amortiguación de estas presiones resultarían en la expansión aún mayor de las masas forestales de la especie en España.

Si bien las particulares condiciones climáticas de las altas cumbres de los macizos de las Nieves y Grazalema, al igual que el resto de los enclaves naturales de la distribución de $A$. pinsapo, sugieren poco probable la expansión de la especie de manera natural a otras zonas de bajas elevaciones aledañas. Los resultados de la plantación de A. pinsapo analizada en este trabajo podrían servir como evidencia alternativa para considerar su potencial expansión fuera del rango natural de distribución de la especie. Las condiciones ambientales en las que el crecimiento es factible en Valdivia, con tasas iguales o aún mayores que en su hábitat original, ocurren también en zonas de España fuera de su hábitat natural. Este estudio demuestra que las condiciones existentes en el arboreto de Isla Teja, en Valdivia, son favorables para el desarrollo de la especie. El clima lluvioso y de temperaturas templadas permite al pinsapo habitar en cotas bajas, próximas al nivel del mar, lo que extiende más aún las regiones habitables por la es- 
pecie, acotada hasta ahora a elevaciones por encima de los $550 \mathrm{msnm}$ (Soto et al. 2001). Por su parte, las condiciones encontradas en Valdivia son similares a las que se dan en una amplia zona de la costa norte de España, y cuya potencialidad no estaba considerada.

Sin embargo, la importancia que tiene la temperatura sobre el crecimiento radial, y su relación con la insolación, podría ser un factor limitante para el crecimiento de la especie en localidades con insolaciones inferiores a las de Valdivia, es decir, por debajo de las 1.700 horas de sol al año. Nuevos ensayos de establecimiento de este abeto en distintas condiciones ambientales darán respuestas a estas interrogantes. Por su parte, nuevos estudios dendrocronológicos en su hábitat natural son imperativos para conocer las respuestas actuales de la especie frente a los cambios en el clima que experimenta la península Ibérica. Ello permitirá delinear de mejor forma las proyecciones de la distribución de uno de los abetos más amenazados del mundo.

\section{CONCLUSIONES}

La primera evaluación de una plantación de $A$. pinsapo en Chile establecida hace 43 años en la ciudad de Valdivia, presentó un crecimiento radial levemente superior a los individuos de crecimiento más acelerado de los pinsapares de las cordilleras béticas de la península ibérica. Lo anterior comprueba la hipótesis que la especie presenta un buen desarrollo en el clima templado lluvioso que caracteriza a la selva valdiviana, posiblemente por su menor oscilación térmica y suelos más profundos respecto a las montañas donde la especie crece en forma natural en la península ibérica. A diferencia de la población de España, donde presenta limitaciones de suelos delgados, competencia y menor pluviosidad, en Valdivia, la principal limitante para el desarrollo de la especie son las horas de insolación restringidas por la nubosidad en esta ciudad.

El incremento radial anual de la especie estuvo relacionado con la precipitación de invierno del año corriente y la temperatura de verano del año previo. En general, la madera temprana se relacionó con la variabilidad del clima del año previo, mientras la madera tardía se relacionó con la variabilidad del clima del año corriente.

El desempeño de la especie en Valdivia sugiere que esta ciudad y posiblemente otras zonas de esta región de Chile y de características climáticas similares en España, podrían ser alternativas viables para la conservación exsitu de la especie.

\section{AGRADECIMIENTOS}

Los autores agradecen al arboreto de la Universidad Austral de Chile por las facilidades para el desarrollo de este estudio. También agradecen a Francisca Veas por su apoyo en la elaboración de figuras. Al Proyecto FONDECYT Iniciación $\mathrm{N}^{\circ} 11161061$, FONDECYT №1181956 y al Centro de Ciencia del Clima y la Resiliencia (CR)2 (FONDAP 15110009).

\section{REFERENCIAS}

Alba-Sánchez F, JA López-Sáez, BBD Pando, JC Linares, D Nieto-Lugilde, L López-Merino. 2010. Past and present potential distribution of the Iberian Abies species: a phytogeographic approach using fossil pollen data and species distribution models. Diversity and Distributions 16(2): 214-228. DOI: https://doi.org/10.1111/j.1472-4642.2010.00636.x.

Blasing TJ, AM Solomon, DN Duvick. 1984. Response functions revisited. Tree-Ring Bulletin 44: 1-15. Consultado 17 de abril de 2019. Disponible en https://repository.arizona.edu/ handle/10150/261260.

CONAMA (Comisión Nacional del Medio Ambiente, CL). 2006. Estudio de la variabilidad climática en Chile para el siglo XXI. Informe Final. Consultado 10 de mayo 2018. Disponible en http://dgf.uchile.cl/PRECIS/articles-39442_pdf Estudio texto.pdf.

Cook ER. 1985. A time series analysis approach to tree ring Standardization. PhD. Dissertation. Tucson, AZ, USA. School of Renewable Natural Resources, University of Arizona. 171 p.

Esteban LG, P De Palacios, LRL Aguado. 2010. Abies pinsapo forests in Spain and Morocco: threats and conservation. Oryx 44(2): 276-284. DOI: https://doi.org/10.1017/ $\underline{\mathrm{S} 0030605310000190}$

Fritts HC. 1976. Tree rings and climate. Academic Press, New York. 576 pp.

Génova M. 2007. El crecimiento de Abies pinsapo y el clima de Grazalema: aportaciones dendroecológicas. Investigación agraria: Sistemas y Recursos Forestales 16(2): 145-157. Consultado 17 de abril de 2019. Disponible en https://recyt. fecyt.es/index.php/IA/article/view/2214/1623.

Génova M. 2013. Dendroclimatología de Abies pinsapo. In López-Quintanilla eds. Los pinsapares en Andalucía (Abies pinsapo Boiss.): conservación y sostenibilidad en el siglo XXI. Córdoba, España. Servicio de Publicaciones Universidad de Córdoba. p. 227-233.

Génova M, E Muñoz. 2005. Caracterización de los pinsapares desde la perspectiva dendroecológica: Determinación de las relaciones entre el crecimiento y el clima. Abstracts IV Congreso Forestal Español. Consultado 17 de abril de 2019. Disponible en http://webcache.googleusercontent. com/search?q=cache:8XLZCRD1qo4J:secforestales.org/ publicaciones/index.php/congresos forestales/article/dow nload $/ 16050 / 15893 /+\& \mathrm{~cd}=3 \& \mathrm{hl}=\mathrm{es} \& \mathrm{ct}=\mathrm{clnk} \& \mathrm{gl}=\mathrm{cl}$.

Gómez-Zotano J, J Alcántara-Manzanares, JA Olmedo-Cobo, E Martínez-Ibarra. 2015. La sistematización del clima mediterráneo: identificación, clasificación y caracterización climática de Andalucía (España). Revista de Geografía Norte Grande (61): 161-180. DOI: http://dx.doi.org/10.4067/ S0718-34022015000200009.

González-Reyes Á, AA Muñoz. 2013. Cambios en la precipitación de la ciudad de Valdivia (Chile) durante los últimos 150 años. Bosque 34(2): 200-213. DOI: http://dx.doi. org/10.4067/S0717-92002013000200008.

Holmes RL. 1983. Computer-assisted quality control in tree-ring dating and measurement. Tree-ring Bulletin 43(1): 69-78.

Huber A. 1970. Diez años de observaciones climatológicas en la estación Teja-Valdivia (Chile), 1960-1969. Valdivia, Uni- 
versidad Austral, Instituto de Geología y Geografía. 46P.

Latorre AV, BC Artero. 2002. La flora y el paisaje vegetal de la provincia de Málaga: importancia y conservación. Jábega 90: 25-39.

Linares JC, JJ Camarero, A Delgado-Huertas, JA Carreira. 2013. Efectos de los cambios de clima y usos del territorio sobre la dinámica y el crecimiento de los bosques de Abies pinsapo en las últimas décadas. In Cerrillo RN. Los pinsapares en Andalucía (Abies pinsapo Boiss.): conservación y sostenibilidad en el siglo XXI. Córdoba, España. Servicio de Publicaciones Universidad de Córdoba. p. 401-413.

Linares JC, JA Carreira. 2006. El pinsapo, abeto endémico andaluz. O ¿Qué hace un tipo como tú en un sitio como éste?. Revista Ecosistemas 15(3).

Luebert F, P Pliscoff. 2005. Sobre los límites del bosque valdiviano en Chile. Chloris Chilensis 8(1).

Navarrete A. 2006. Estado de desarrollo ex-situ de quillay (Quillaja saponaria Mol.), keule (Gomortega keule (Mol.) Baillon) y belloto del sur (Beilschmiedia berteroana (Gay) Kosterm.) en Valdivia. Tesis Ingeniero Forestal. Valdivia, Chile. Facultad de Ciencias Forestales, Universidad Austral de Chile. 34 p.

Pérez FF, R Boscolo. 2010. Climate in Spain: Past, present and future. Regional climatic change assessment report. Consultado 17 abr. 2019. Disponible en http://www.clivar.org/ sites/default/files/documents/CLIVAR_Spain_2010_0.pdf.

Pompa-García M, JJ Camarero-Martínez. 2015. Potencial den- droclimático de la madera temprana y tardía de Pinus cooperi Blanco. Agrociencia 49(2): 177-187. Consultado 17 de abril 2019. Disponible en http://digital.csic.es/bitstream/10261/117630/1/Pompa camarero potencial_pinus_coperi_2015.pdf.

Schulman E. 1956. Dendroclimatic changes in semiarid America. Dendroclimatic changes in semiarid America. Tucson, AZ, USA. University of Arizona Press. p. 142.

Soto D, JG Viñas, EP Buj. 2001. Expansión naturalizada de Abies pinsapo en España. Congresos Forestales. Consultado 17 abr. 2019. Disponible en http://secforestales.org/ publicaciones/index.php/congresos forestales/article/ view/15908/15751.

Uggla C, E Magel, T Moritz, B Sundberg. 2001. Function and dynamics of auxin and carbohydrates during earlywood/ latewood transition in Scots pine. Plant Physiology 125(4): 2029-2039. DOI: https://doi.org/10.1104/pp.125.4.2029.

Van der Maaten E. 2012. Climate sensitivity of radial growth in European beech (Fagus sylvatica L.) at different aspects in southwestern Germany. Trees 26(3):777-788. DOI: https:// doi.org/10.1007/s00468-011-0645-8.

Wigley TML, KR Briffa, PD Jones. 1984. On the average value of correlated time series, with applications in dendroclimatology and hydrometeorology. Journal of Climate and Applied Meteorology 23: 201-213. DOI: https://doi. org/10.1175/1520-0450(1984)023<0201: OTAVOC $>2.0$. $\mathrm{CO} ; 2$. 